\title{
Efficient determination of thermodynamic properties from a single simulation
}

\author{
J. M. Rickman ${ }^{\text {a) }}$ and D. J. Srolovitz \\ Department of Materials Science and Engineering, The University of Michigan, Ann Arbor, \\ Michigan 48109
}

(Received 12 July 1993; accepted 5 August 1993)

\begin{abstract}
A method for calculating the density of states of a system directly from its trajectory in phase space is described. As a specific example, the method is applied to the Monte Carlo simulation of a two-dimensional Ising model. The energy distribution function is calculated from the density of states and the associated Helmholtz free energy per spin is calculated for various system sizes and temperatures and shown to be in excellent agreement with the exact results.
\end{abstract}

\section{INTRODUCTION}

Since the thermodynamics of any system may be obtained directly from the free energy or partition function, the determination of these quantities is central to much of statistical physics. While thermodynamic derivatives of the free energy, such as the internal energy or magnetization, may be expressed in terms of ensemble averages of dynamical variables normally accessible by computer simulation, this is not true of the free energy itself. According to Binder, ${ }^{1}$ the "Monte Carlo method yields information in quantities which are thermal averages of observables, but it does not yield any estimates for the partition function $Z$ itself. As a result, while the internal energy is easily calculated, neither the free energy $F=-k_{B} T \ln Z$ nor entropy are obtained directly." In the present paper, we demonstrate that the partition function and therefore the "absolute" free energy may be calculated directly from a simulation and propose a simple, efficient method for doing so.

Several methods to indirectly extract free energies from computer simulations have been proposed. ${ }^{2-6}$ The now standard approach to this problem has been to relate quantities such as the free energy or entropy of the system of interest to that of a conveniently chosen reference system by the construction of an integration path between the systems. ${ }^{2,4}$ While methods based upon this concept have had some successes, the extremely large computational demands inherent to these indirect approaches have limited their applicability to a small number of very simple systems. However, as the entropy is related to the volume of the allowed region of phase space by Boltzmann's equation, it should also be possible to calculate the entropy directly from a detailed knowledge of the trajectory of a system (the set of states realized in a simulation).

$\mathrm{Ma},{ }^{7}$ for example, developed a method to calculate the entropy from a trajectory based on the assumption that the states comprising the trajectory can be divided into groups of states which are each uniformly distributed in regions of phase space. The phase space volume of these regions, and hence the entropy, can be determined by counting the numbers of pairs of states in a region that coincide. This

\footnotetext{
a)Permanent address: Department of Materials Science and Engineering, Lehigh University, Bethlehem, PA 18015.
}

method has been applied, with some success, to calculate the entropy of small, 1-D Ising models consisting of up to 16 spins and the entropy of a 2-D Sherrington-Kirkpatrick spin glass at low temperatures. ${ }^{8}$ While this approach is very appealing, its principal drawbacks are that it is not very accurate (the calculated spin entropy differs from the exact value by approximately $5 \%$ ) and that it is applicable only to small systems or low temperatures.

Other approaches, such as histogram analyses ${ }^{9}$ and cumulant expansions ${ }^{10}$ have permitted the calculation of free energy and entropy over appreciable ranges of parameter space from a single simulation. Such approaches effectively determine free energy and entropy differences from trajectories since an energy histogram is a summary of the fraction of time spent in various regions of phase space. Unfortunately, since simulations rarely access the very high and low tails of the energy histogram, these methods can only explore a limited range of parameter (e.g., temperature and pressure) space. Whereas the histogram and cumulant expansion approaches often require the introduction of a reference state to determine free energies, it is advantageous to obtain the "absolute" free energy and its generating function, the partition function, directly from the simulation.

In this paper we show that the density of states may be calculated directly from a trajectory in a simulation. The "absolute" free energy, the entropy, and all other thermodynamic quantities can be directly determined from the density of states. This method is much more accurate and efficient than the coincidence-counting method, and its implementation is straightforward. Unlike numerical methods for calculating the density of states from several artificially constrained systems with related Hamiltonians, ${ }^{11}$ our method is based on a direct analysis of the trajectory of the unconstrained system of interest. The direct determination of the density of states in a simulation at one temperature has the additional advantage that it allows one to calculate properties over a wide range of temperature based upon a single simulation at one temperature. As a specific example of this general method, we explicitly analyze the well-known case of a 2-D Ising model. 


\section{PROCEDURE}

Consider a system in thermodynamic equilibrium at a temperature $T$ interacting with a heat reservoir. The average properties of this system, such as its specific heat or magnetization, can be calculated from a series of configurations (a trajectory) generated, for example, by using a canonical Monte Carlo simulation. These configurations can be sorted into groups with the same energy, $E$. Each group corresponds to a region in phase space consisting of $g(E)$ (the density of states) total states, and it is assumed that the generated configurations within any group are randomly distributed in the associated region of phase space. The thermodynamic properties of this system at inverse temperaturc $\beta=1 / k_{B} T$ can then be calculated from its partition function

$$
Z(\beta)=\sum_{E} g(E) e^{-\beta E},
$$

where the summation is over the discrete set of energies available to the system. Hence, all thermodynamic properties can be calculated once the density of states $g(E)$, and hence the partition function $Z$, is known.

In order to calculate $g(E)$ directly from a simulation, we have found the following approach to be both efficient and easy to implement in a Monte Carlo simulation. First, from a knowledge of the possible excitation energies for the system under consideration, identify a special subset of $\bar{g}(E)$ states for each energy $E$. This subset is simply a convenient set of easily identifiable states. For example, in the case of the one-dimensional, ferromagnetic Ising model subject to periodic boundary conditions, states corresponding to a given excitation energy can be classified in terms of the number of bonds between unlike spins, where each such bond contributes $2 J$ ( $J$ is the energy parameter) to the excitation energy. Thus, a special subset of states having energy $4 n J$, where $n$ is a positive integer, consists of $n$ isolated "down" spins such that no two "down" spins are nearest neighbors. This can be created by placing the $n$ "down" spins on the even-numbered lattice sites and placing "up" spins on all of the remaining sites. There are, of course, other ways to choose special subsets. It is convenient to choose special subsets based on some simple rules, as in the example above. It is essential, however, that the precise number of states within a special subset be calculable in order to implement this information into the scheme described below.

After making this identification, group the configurations in a generated trajectory by energy and examine the set of states in each group recording whether or not a state is contained in the special subset of states for that group. This operation can be performed during the course of the simulation by simply comparing a given configuration with those in the special subset. For example, in the case of the one-dimensional Ising model discussed above, one could simply ask whether a generated configuration consists of "down" spins on even-numbered sites and "up" spins on the remaining even-numbered sites and on all of the odd- numbered sites. If the answer is yes, then this configuration belongs to the special subset.

The fraction of realized configurations of energy $E$ that fall within the subset is the probability function $f(E)$. Since generated configurations are randomly distributed in each group $g(E)=\bar{g}(E) / f(E)$. That is, $f(E)$ is an unbiased estimator of the fraction of states in the special subset. Therefore a determination of $f(E)$ for all important excitation energies directly from simulation yields $g(E)$ and, by using Eq. (1), $Z$. The only assumption that is made in determining $Z$ by this prescription is that configurations corresponding to the same energy are randomly distributed in their associated region of phase space.

Upon obtaining $f(E)$, and hence, $g(E)$ from configurations generated in a simulation at one temperature, properties at other temperatures can be determined by using Eq. (1) without resorting to further simulations. Thus, for example, the "absolute" free energy, $F=-k_{B} T \ln Z$, can be obtained over a wide range of temperature without the introduction of a reference state. The ensemble average of a quantity $X,\langle X\rangle$, can be directly calculated from $g(E)$ as $\langle X\rangle=\Sigma_{E} X g(E) e^{-\beta E} / Z(\beta)$, where $Z(\beta)$ is defined in terms of $g(E)$ in Eq. (1).

Further, if one is only interested in determining the partition function, it is not necessary to determine $g(E)$ for all energies $E$ in a trajectory. In fact, it is only necessary to calculate $g(E)$ for a specific energy $\hat{E}$ since the fraction of time $p(\hat{E})$ that the system spends with energy $\hat{E}$ is given by

$$
p(\hat{E})=g(\hat{E}) e^{-\beta \hat{E}} / Z(\beta) .
$$

So, $Z(\beta)$ is then determined once $p(\hat{E})$ and $g(\hat{E})$ are obtained from a simulation. As a practical matter, it is necessary to choose an $\hat{E}$ that is sampled often enough in a simulation in order to accurately determine $p(\hat{E})$ and $g(\hat{E}) . g(\hat{E})$ can, of course, be calculated by identifying a convenient special subset of states having energy $\hat{E}$ and then using the previously described technique. The key to a successful implementation of this counting method is clearly the identification of easily distinguishable subsets in which $\bar{g}(E)$ is large. This second approach will be implemented below in order to obtain the free energy of large systems.

\section{EXAMPLE: ISING MODEL}

In order to make these ideas more concrete, we consider, as a specific example, a ferromagnetic 2-D Ising model having $N$ spins with nearest-neighbor interaction energy $J$ on a square lattice subject to periodic boundary conditions. For this model, the possible excitation energies are given by $\epsilon=4 p J$, where $p$ is a non-negative integer and $p \neq 1$. The identification of special energy subsets is facilitated by dividing these excitation energies into two classes. In the first class, a subset of states having excitation energy $\epsilon_{n}$ consists of $n$ isolated spins with $s=-1$ (or +1 ) such that no two spins with $s=-1$ (or +1 ) are nearest neighbors. The energy of a subset with $n$ isolated spins is easily calculated by noting that a broken bond has excitation 
TABLE I. The probability function $f(E)$ and the number of states $\bar{g}(E)$ in the special subsets of states for several excitation energies for a $N=36$ Ising model with $T=3.20 J / k_{B}$. The density of states can be found from $g(E)=\bar{g}(E) / f(E)$.

\begin{tabular}{|c|c|c|c|c|c|c|}
\hline & $24 J$ & $36 J$ & $48 J$ & $60 \mathrm{~J}$ & $72 J$ & $76 J$ \\
\hline $\begin{array}{l}\bar{g}(E) \\
f(E)\end{array}$ & $\begin{array}{c}9816 \\
2.783 \times 10^{-1}\end{array}$ & $\begin{array}{c}307008 \\
7.912 \times 10^{-2}\end{array}$ & $\begin{array}{c}505584 \\
1.934 \times 10^{-3}\end{array}$ & $\begin{array}{c}5203728 \\
1.135 \times 10^{-3}\end{array}$ & $\begin{array}{c}961832 \\
4.236 \times 10^{-5}\end{array}$ & $\begin{array}{c}4467168 \\
2.936 \times 10^{-4}\end{array}$ \\
\hline
\end{tabular}

energy $2 J$ and each isolated spin creates four broken bonds, i.e., $\epsilon_{n}=8 n J$. In the second class, a subset of states having energy $\epsilon_{m}$ consists of a single nearest-neighbor pair of spins with $s=-1$ (or +1 ), $m$ isolated spins with $s=-1$ (or +1 ) and $N-m-2$ spins with $s=+1$ (or -1 ) such that no two spins, other than the pair, are nearest neighbors. The energy of this subset is calculated by observing that the nearest-neighbor pair of spins creates six broken bonds and the other $m$ isolated spins again each create four broken bonds, i.e., $\epsilon_{m}=12+8 m J$. This scheme is particular convenient as the configurations associated with each class are easily visualized as a collection of independent excitations. Very many other choices of subsets are, of course, also possible.

The number of states $\bar{g}(E)$, where $E=-2 N J+\epsilon_{n}$ or $-2 N J+\epsilon_{m}$, were readily obtained from simple counting program and combinatorial analysis. The procedure to obtain $g(E)$ directly from simulation, then is to examine the configurations generated in a Monte Carlo simulation at temperature $T$ and record whether they are elements of the special subsets, thereby determining the probability function $f(E)$ and, hence, $g(E)$. The simplicity of the classification scheme permits easy subdivision of the total set of configurations into subsets.

The method was first applied to an $N=36$ Ising spin system on a square lattice at $T=3.20 \mathrm{~J} / k_{B}$. The trajectory consisted of approximately $2.40 \times 10^{7}$ Monte Carlo steps sampled from a total of $1.20 \times 10^{8}$ in four independent runs. The values of $f(E)$, along with $\bar{g}(E)$ as calculated from the counting program, for several excitation energies $\epsilon$ are summarized in Table I. As is evident from the table, even though $g(E)$ can be quite large for some energies

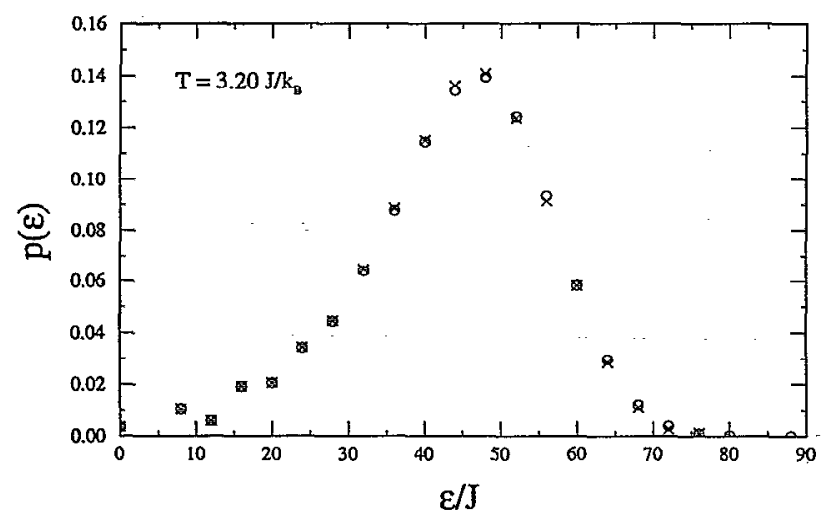

FIG. 1. The energy histograms, $p(\epsilon)$, for $N=36$ spins as obtained in simulations at $T=3.20 \mathrm{~J} / \mathrm{k}_{B}(O)$ and as calculated using the density of states $g(E)$ determined at $T=3.20 \mathrm{~J} / k_{B}(X)$. $\left(\sim 10^{11}\right)$, it can be accurately determined by regarding it as a product of $\vec{g}(E)$ and $1 / f(E)$. Figure 1 (a) shows the histogram $p(\epsilon)$ obtained in a simulation at $T=3.20 \mathrm{~J} / \mathrm{k}_{B}$ and that predicted by using the calculated density of states, $p(E)=g(E) e^{-\beta E} / Z(\beta)$. The histogram $p(\epsilon)$ for $T=2.40 \mathrm{~J} / \mathrm{k}_{B}$, as obtained in a simulation at that temperature, and as predicted by using the density of states determined from the $T=3.20 \mathrm{~J} / \mathrm{k}_{B}$ simulation are compared in Fig. 2. The complex behavior of this curve at small $\epsilon / J$ is attributable to the finite lattice size and the fact that $T=2.40 \mathrm{~J} / k_{B}$ is very near the critical temperature. As is apparent from Figs. 1 and 2, the agreement between the two histograms for each temperature is excellent, and, consequently, thermodynamic averages can be accurately calculated over a wide range of temperature. The Helmholtz free energy per spin and the entropy per spin, calculated from the partition function in Eq. (1) for $T=3.20 \mathrm{~J} / k_{B}$, are $F / N J=-2.5638 \pm 0.0008$ and $S / N k_{B}=0.5532$ \pm 0.0014 , respectively. The free energy as a function of temperature over a wide range of temperature, as calculated from Eq. (1), is shown in Fig. 3 along with the exact solution for the finite size, 2-D Ising model. ${ }^{12}$ The agreement between these thermodynamic functions and their exact values is excellent, thereby indicating the power of the present approach.

For large systems, it becomes increasingly difficult to calculate the density of states $g(E)$ for all excitation energies. This is because some excitations, particularly in the wings of the histogram, are sampled comparatively rarely and because the counting algorithm becomes less efficient for large excitation energies, as evidenced by small values of $f(E)$-see Table I. Nevertheless, it is still possible to

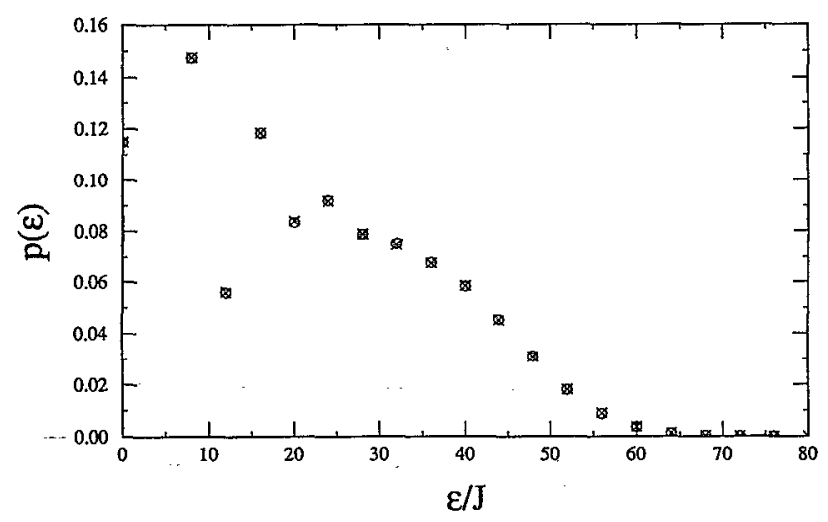

FIG. 2. $p(\epsilon)$ as obtained at $T=2.40 J / k_{B}(O)$ and calculated by using the $g(E)$ determined at $T=3.20 J / k_{B}(X)$. The number of spins $N=36$. 


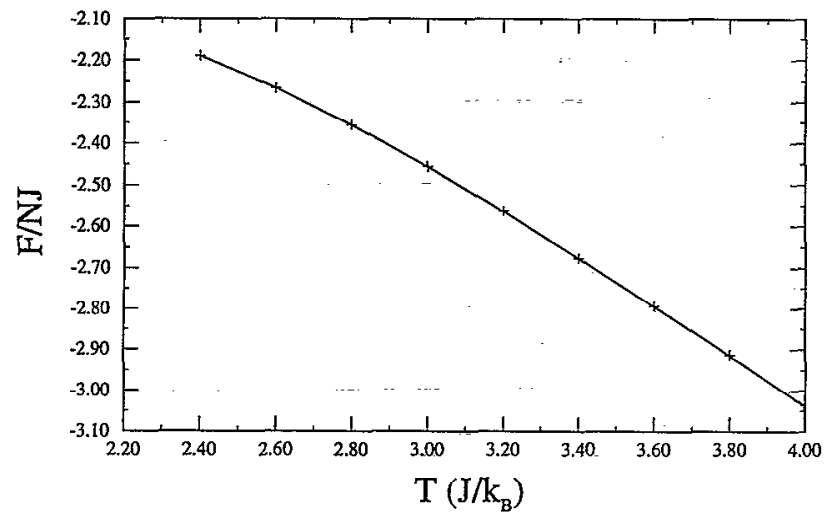

FIG. 3. The free energy per spin $F / N J$ vs temperature, $T$, for $N=36$ as calculated using the density of states $(+)$. The exact result is shown as a solid line.

accurately calculate the free energy by using Eq. (2). To do this, choose an energy $\hat{E}$ that is not far out in the wings of the histogram and obtain both $g(\hat{E})$ and $p(\hat{E})$ from the trajectory generated in the simulation. The "absolute" free energy per spin $F / N J$ was determined for systems at $T=3.20 J / k_{B}$ with $N=16,25$, and 36 calculating $g(E)$ and using Eq. (1) and for larger systems with $N=49,64$, and 100 by obtaining both $g(\hat{E})$ and $p(\hat{E})$ for a single $\hat{E}=-2 N J+48 J$. The results of these calculations are shown in Fig. 4 along with the exact results. ${ }^{12}$ These results indicate that Eq. (2) can be used to accurately determine the partition function and free energy directly from a simulation provided that $g(\hat{E})$ is known for a single, relatively large energy $\hat{E}$.

Figure 4 shows that the calculated free energy per spin is within $0.06 \%$ of the infinite size system value. Therefore, we conclude that while there are some size limitations to the counting scheme presented here, the method for determining thermodynamic properties described is sufficient to obtain accurate data on effectively infinite systems. In short, if the length scale of the system is much greater than

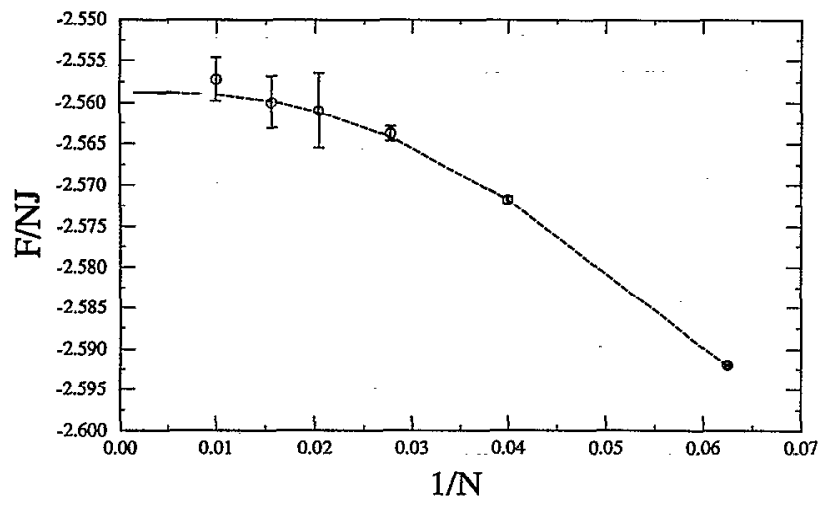

FIG. 4. The free energy per spin $F / N J$ vs $1 / N$ for $N=16,25,36,49,64$, and 100. For $N \leqslant 36, F / N J$ was determined from the entire histogram generated by the calculated density of states, while for $N>36, F / N J$ was calculated by using Eq. (2) with $E=E_{0}+48 J$. The exact result is shown as a solid line. Error bars were determined from the variance in $f(E)$ as calculated from independent runs. the correlation length $\xi$, then the system can be regarded as a collection of nearly independent, smaller subsystems ${ }^{13}$ and the extrapolation to large system size is justified. The application of our approach to more complex systems with multiple time scales, as in the case of polymers, is, however, currently problematic in practice. Although it is possible to obtain information on the density of states, following the long-time trajectories in these systems is impractical.

\section{CONCLUSIONS}

In the present paper, we develop a new method for determining the density of states, partition function, free energy, and all other thermodynamic properties directly from a simulation. The partition function is, of course, the central thermodynamic quantity of interest since other thermodynamic quantities are derivable from it. The density of states results determined from a single simulation at a single temperature may be used to predict the thermodynamic properties of the system at any temperature. Therefore, this approach may be used to predict the properties of the system near critical points, based on simulations at $T$ far from $T_{c}$. As an example, this procedure as applied to a two-dimensional Ising model. The free energy vs temperature of this model was determined from a single simulation and was shown to agree with the exact result to within $0.02 \%$. A method for obtaining the free energy for large system sizes was described and shown to agree with the exact, infinite system size results to within $0.06 \%$.

The application of our procedure to systems with continuous potentials is somewhat more involved. In these cases it is more difficult to identify a subset of states with a given energy. One possible approach to overcoming this problem is to record a series of configurations and their corresponding energies during the course of a simulation, and then perform a second simulation wherein a comparison is made between configurations generated in the second simulation and those generated in the first. This will undoubtedly require long simulations in order to generate a large number of states for the comparison. The application of this approach to determine accurate thermodynamic data from simulation trajectories in other discrete models and in fluids is the subject of ongoing research.

\section{ACKNOWLEDGMENTS}

The authors gratefully acknowledge many helpful conversations with Dr. Jorge Viñals and the support of the Defense Advanced Research Projects Agency (DARPA) and the Office of Naval Research under Contract No. N00014-91-J-4019.

\footnotetext{
${ }^{1}$ K. Binder, J. Comp. Phys. 59, 1 (1985).

${ }^{2}$ D. Frenkel and A. J. C. Ladd, J. Chem. Phys. 81, 3188 (1984).

${ }^{3}$ K. K. Mon, Phys. Rev. Lett. 54, 2671 (1985).

${ }^{4}$ J. Q. Broughton and X. P. Li, Phys. Rev. B 35, 9120 (1987).

${ }^{5}$ S. R. Phillpot and J. M. Rickman, J. Chem. Phys. 94, 1454 (1991).
} 
${ }^{6}$ R. LeSar, R. Najafabadi,and D. J. Srolovitz, Phys. Rev. Lett. 63, 624 (1989).

${ }^{7}$ S.-K. Ma, J. Stat. Phys. 26, 221 (1981).

${ }^{8}$ S.-K. Ma and M. Payne, Phys. Rev. B 24, 3984 (1981).

${ }^{9}$ I. R. MacDonald and K. Singer, Discuss. Faraday Soc. 43, 40 (1967);

A. M. Ferrenberg and R. H. Swendsen, Phys. Rev. Lett. 61, 2635 (1988).
${ }^{10}$ J. M. Rickman and S. R. Phillpot, Phys. Rev. Lett. 66, 349 (1991).

${ }^{11}$ G. Bhanot, R. Salvador, S. Black, P. Carter, and R. Toral, Phys. Rev. Lett. 59, 803 (1987).

${ }^{12}$ L. Onsager, Phys. Rev. 65, 117 (1944); A. E. Ferdinand and M. E. Fisher, Phys. Rev. 185, 832 (1969).

${ }^{13}$ S.-K. Ma, Statistical Mechanics (World Scientific, Philadelphia, 1985). 